\title{
Do Neurocritical Care Units Save Lives? Measuring The Impact of Specialized ICUs
}

\author{
Andreas H. Kramer • David A. Zygun
}

Published online: 18 March 2011

(C) Springer Science+Business Media, LLC 2011

A variety of organizational models have been used to deliver care to critically ill patients with neurologic disorders. Primary care may be provided by general intensivists, who often rely heavily on consultative support from neurosurgeons and neurologists. This approach is especially common in Australia, New Zealand, Canada and some European countries. Intensive care units (ICUs) organized in this fashion are usually "closed", meaning that admissions and discharges are largely the responsibility of the attending intensivist. In this case, there are typically daily multidisciplinary rounds with a single team of clinicians. An alternative method is for primary care to be delivered by neurosurgeons or neurologists, in this case depending greatly on consultative input from various subspecialists. The corresponding ICUs are frequently "open"; that is, at any given time, there may be multiple attending physicians with patients admitted under their care, each of which, in turn, has numerous consultants involved. This approach has, historically, been the most common to be used in the United States.

Electronic supplementary material The online version of this article (doi:10.1007/s12028-011-9530-y) contains supplementary material, which is available to authorized users.

A. H. Kramer ( $\square)$

Departments of Critical Care Medicine \& Clinical

Neurosciences, Hotchkiss Brain Institute, Foothills Medical

Center, University of Calgary, Calgary, Canada

e-mail: andreas.kramer@calgaryhealthregion.ca

D. A. Zygun

Departments of Critical Care Medicine, Clinical Neurosciences

\& Community Health Sciences, Hotchkiss Brain Institute,

Foothills Medical Center, University of Calgary,

Calgary, Canada
The skill set of ICU bedside nurses and ancillary health professionals (e.g., respiratory therapists, pharmacists, social workers, and rehabilitation staff) may also vary. In "general ICUs", these individuals are usually well-trained in the provision of physiologic support, especially to patients with multi-organ failure; however, specific nuances that are important to neurocritical care patients may sometimes be under-recognized. In contrast, in specialized ICUs, nurses are specifically trained to detect and treat neurologic deterioration in a timely fashion; in this case, however, there may be less experience in the management of systemic complications.

As a specialty, neurocritical care seeks to combine the advantages of each of these preceding models. Neurointensivists and neurocritical care nurses are content experts in both critical care and neurologic disorders. These individuals are trained especially to recognize when brain- and spinalcord-injured individuals have unique physiologic considerations in relation to other critically ill patients. Thus, their presence influences the "ICU culture" to become highly focused on neuroprotection; treatment targets (e.g., blood pressure or temperature) are consistently chosen with the aim of preventing "secondary injury", increasingly with the aid of advanced multi-modal monitoring. Furthermore, these clinicians are well equipped to deal with the non-neurologic organ dysfunction that is commonly encountered in critically ill neurologic patients [1].

Allied health professionals in neurocritical care units become familiar with the specific implications of the therapeutic interventions that they provide. For example, respiratory therapists are keenly aware that even minor variations in the partial pressure of carbon dioxide can have major implications on cerebral blood flow and intracranial pressure; "neuro-pharmacists" become particularly knowledgeable about the neurologic complications of 
commonly used drugs; and social workers gain experience with the unique needs experienced by families of braininjured patients. Rounds are usually multidisciplinary, thereby facilitating healthy interactions between staff and ensuring that all perspectives are heard. An increasing proportion of neurocritical care units are closed or "semiclosed", which enhances communication and helps make certain that patients receive (as per the Society of Critical Care Medicine motto) "the right care, right now".

In some ways, it would seem obvious that a team of dedicated neurocritical care specialists would represent the optimal model for the delivery of care to critically ill neurologic patients. However, because of the complexities of critical care, it is difficult to conclusively demonstrate efficacy, even for interventions that intuitively make sense. A good example of this is the development of medical emergency teams (METs) to provide timely care to deteriorating, hospitalized patients outside of the ICU. Although few clinicians doubt that METs are of benefit, results of clinical studies have been mixed; efficacy could not be definitively proven in a large, cluster randomized controlled trial (RCT) [2].

In the current edition of Neurocritical Care, Samuels et al. [3] have provided the latest in a growing number of studies demonstrating improvements in outcomes when a multidisciplinary neurocritical care model is embraced. As in other similar preceding articles, the study was a singlecenter, retrospective comparison of outcomes during two sequential time periods, before and after the appointment of a neurointensivist-led team, consisting also of nurse practitioners, a pharmacist, respiratory therapists, and a dietician. Although no differences in mortality were observed over time, patients in the more recent cohort were significantly more likely to be discharged home, rather than to a rehabilitation or long-term care facility. This effect was observed across all subgroups of SAH severity, but was most pronounced among low grade (Hunt-Hess grade I-III) patients. Unfortunately, measures of functional neurologic recovery (e.g., modified Rankin scores) were not determined.

To integrate the findings of this study with previously published research, we systematically searched the literature (see Electronic Supplementary Material). We identified 12 studies, involving 24,520 patients, which presented original data comparing models of care for critically ill neurologic patients [3-14]. Four studies assessing subgroups derived from previously published data were excluded (see Electronic Supplementary Material) [15-18].

Study characteristics are shown in Table 1. Ten studies were performed at single centers; each of these had a "before and after" design, whereby patients treated using a new model of care were compared with historical controls $[3,4,6-11,13,14]$. Two studies were methodologically stronger, in that they involved multiple centers and compared patients treated in specialized ICUs with concurrent controls cared for in generalized units [5, 12]. Most, but not all, studies performed multivariate analysis, adjusting for at least some potentially confounding variables $[3,5,9,10$, $12,14]$. The "intervention" of interest varied considerably across studies: in most cases, care was provided in a

Table 1 Characteristics of studies comparing models used to deliver care to 24,520 brain-injured patients

\begin{tabular}{llllllll}
\hline Study & Centers & Population & No. pts & Controls & Neuro-intensivists & Risk adjustment & Functional outcomes \\
\hline Warme [4] & Single & TBI & 121 & Historical & No & No & Yes (GOS) \\
Diringer [5] & Multiple & ICH & 1037 & Concurrent & Yes & Yes & No \\
Mirski [6] & Single & ICH & 128 & Historical & Yes & No & No \\
Elf [7] & Single & TBI & 226 & Historical & No & No & Yes (GOS) \\
Patel [8] & Single & TBI & 285 & Historical & Yes & No & Yes (GOS) \\
Varelas [10] & Single & Variable & 2366 & Historical & Yes & Yes & No \\
Suarez [9] & Single & Variable & 2381 & Historical & Yes & Yes & No \\
Lerch [11] & Single & SAH & $59^{\text {a }}$ & Historical & Yes & No & Yes (GOS) \\
Lott [12] & Multiple & IH & 11868 & Concurrent & Unclear Probably variable & Yes & No \\
Josephson [13] & Single & SAH & 512 & Historical & Yes & No & Yes \\
Palminteri ${ }^{\text {[14] }}$ & Single & ICH & 287 & Historical & Yes & No & Yes (unclear) \\
Samuels [3] & Single & SAH & 703 & Historical & Yes & No & \\
\hline
\end{tabular}

GOS Glasgow Outcome Scale, $I C H$ intracerebral hemorrhage, $I H$ "intracranial hemorrhage" (authors did not subdivide further), $I S$ ischemic stroke, $S A H$ subarachnoid hemorrhage, $T B I$ traumatic brain injury

a Authors reported major transition in severity of illness over time and therefore restricted analysis to subgroup of patients with World Federation of Neurological Surgeons grade 4 SAH

b Published only in abstract form 
neurologic ICU, with an attending physician who was a neurointensivist $[3,5,6,8-11,13,14]$; however, in two studies (including the largest), neurologic ICUs were compared with general ICUs, regardless of the particular expertise of clinicians [4, 12]; in another study, there were simply alterations in the process of care, with implementation of protocols designed to prevent secondary brain injury, but no change in personnel [7]. This variability emphasizes that there are potentially various approaches to quality improvement at individual centers.

Two articles did not report hospital mortality rates; correspondence with the authors of each of these enabled us to obtain this information $[6,11]$. Five studies reported detailed outcome information, usually in the form of Glasgow Outcome Scale scores after $\geq 6$ months $[4,7,8$, $11,14]$. Five of the remaining seven studies reported discharge disposition $[3,6,9,10,12]$; for the purposes of this study we defined discharge home (as opposed to a longterm care or rehabilitation facility) as a favorable outcome.

Combining the data from all publications, mortality was clearly lower in specialized neurologic ICUs (Fig. 1; OR $0.78,95 \%$ CI $0.64-0.95, P=0.01)$. However, although almost all individual studies favored dedicated neurocritical care units, there was substantial heterogeneity in the results $\left(\mathrm{I}^{2}=80 \%, \mathrm{Q}=53.7, P<0.0001\right)$. This is to be expected, given the wide variability in the methodology and characteristics of individual studies.

Neurologic outcomes were also improved in neurocritical care units (Fig. 2; OR 1.29, 95\% CI 1.11-1.51, $P=0.001)$. Again, there was a large degree of heterogeneity $\left(\mathrm{I}^{2}=74 \%, \mathrm{Q}=35.0, P<0.0001\right)$. It should also be noted that these findings were derived from unadjusted

\begin{tabular}{lccc} 
Study name & Outcome & \multicolumn{2}{c}{ Yes/Total } \\
\cline { 2 - 3 } & & NCC & $\begin{array}{c}\text { No or } \\
\text { Pre- NCC }\end{array}$ \\
Diringer 2001 & Death & $93 / 266$ & $310 / 771$ \\
Elf 2002 & Death & $9 / 154$ & $22 / 72$ \\
Josephson 2010 & Death & $54 / 296$ & $29 / 216$ \\
Lerch 2006 & Death & $5 / 36$ & $5 / 23$ \\
Lott IH 2009 & Death & $1199 / 5993$ & $1461 / 5875$ \\
Lott IS 2009 & Death & $314 / 1446$ & $661 / 3101$ \\
Mirski 2001 & Death & $15 / 78$ & $18 / 50$ \\
Palminteri 2010 & Death & $36 / 164$ & $30 / 123$ \\
Patel 2002 & Death & $41 / 202$ & $19 / 83$ \\
Samuels 2011 & Death & $98 / 386$ & $85 / 317$ \\
Suarez 2004 & Death & $97 / 1180$ & $127 / 1201$ \\
Varelas 2004 & Death & $116 / 1279$ & $110 / 1087$ \\
Warme 1991 & Death & $23 / 72$ & $20 / 49$ \\
& & $2100 / 11552$ & $2897 / 12968$
\end{tabular}

Fig. 1 Observational studies comparing outcomes between specialized neurologic critical care units and alternative models of care: association with mortality (Note: Lott and colleagues presented data; thus, it is unclear that baseline characteristics of patients between groups were consistently similar. Individual studies generally did not provide sufficient data to combine the results of adjusted analyses into a pooled result.

When we restricted the analysis to studies in which care in the "intervention" group was led by a neurointensivist $[3,5,6,8-11,13,14]$, results were similar. Even though the overall number of patients was considerably smaller, there was still a statistically significant reduction in mortality (OR $0.85,95 \%$ CI $0.74-0.98, P=0.03$ ) and an increment in the proportion of patients with a favorable outcome (OR 1.38, 95\% CI 1.15-1.66, $P=0.0005$ ). Interestingly, the degree of heterogeneity in results was considerably less ( $\mathrm{I}^{2} 9$ and $51 \%$, respectively). Additional benefits ascribed to neurointensivist-led teams have been reported; these include reductions in length of stay [6, 9, 10, 13-16], cost savings [6, 19], less need for ventriculoperitoneal shunts in SAH patients [13], improved documentation [20] and increased organ and tissue donation rates [21]. Delays in transfer of patients from the emergency department to the neurocritical care unit have also been associated with worse outcomes [22].

The validity of any summary of published data is highly dependent on the quality of the individual studies that it combines. Clearly, the design of previously published studies is vulnerable to some bias (Table 1): First, in most cases, the authors had a potential conflict of interest, given that they were presenting data that were essentially measuring their own performance. Accordingly, there exists considerable potential for publication bias, since investigators would perhaps be less likely to publish outcomes

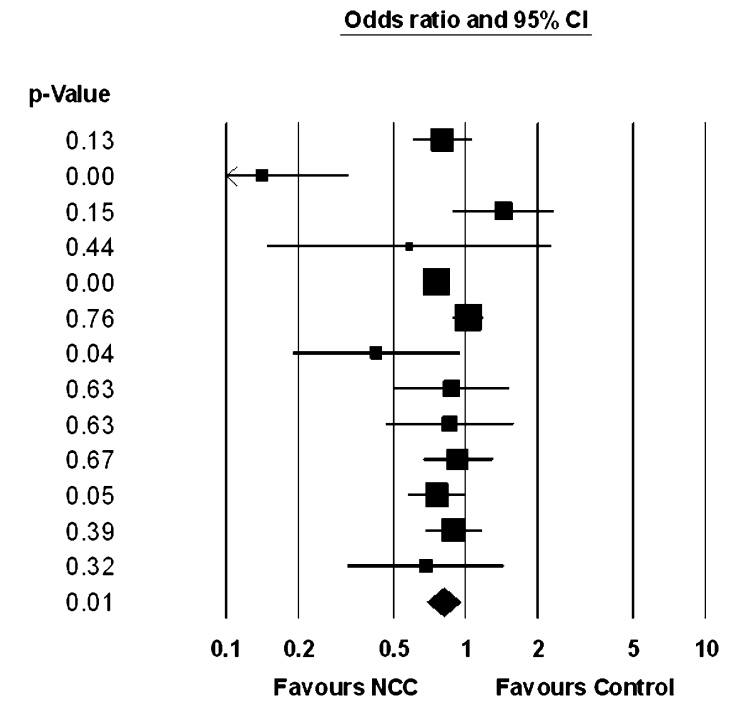

separate data for patients with intracranial hemorrhage and ischemic stroke. The size of squares in Forrest plot is proportional to number of patients in study) 


\begin{tabular}{llccrr} 
Study name & Outcome & \multicolumn{2}{c}{ Yes/Total } \\
\cline { 3 - 5 } & & NCC & $\begin{array}{c}\text { No or } \\
\text { Pre-NCC }\end{array}$ & p-Value \\
Elf 2002 & Favourable Outcome & $120 / 154$ & $34 / 72$ & 0.00 \\
Lerch 2006 & Favourable Outcome & $23 / 36$ & $10 / 23$ & 0.13 \\
Lott IH 2009 & Favourable Outcome & $2883 / 5993$ & $2416 / 5875$ & 0.00 \\
Lott IS 2009 & Favourable Outcome & $538 / 1446$ & $1133 / 3101$ & 0.66 \\
Mirski 2001 & Favourable Outcome & $54 / 78$ & $24 / 50$ & 0.02 \\
Palminteri 2010 & Favourable Outcome & $102 / 164$ & $75 / 123$ & 0.83 \\
Patel 2002 & Favourable Outcome & $134 / 202$ & $46 / 83$ & 0.08 \\
Samuels 2011 & Favourable Outcome & $141 / 386$ & $80 / 317$ & 0.00 \\
Suarez 2004 & Favourable Outcome & $668 / 1180$ & $650 / 1201$ & 0.22 \\
Varelas 2004 & Favourable Outcome & $763 / 1279$ & $562 / 1087$ & 0.00 \\
Warme 1991 & Favourable Outcome & $35 / 72$ & $16 / 49$ & 0.08 \\
& & $5461 / 10990$ & $5046 / 11981$ & 0.00
\end{tabular}

Fig. 2 Observational studies comparing outcomes between specialized neurologic critical care units and alternative models of care: association with favorable outcomes (Note: Lott and colleagues

that did not show the results they were hoping for. However, when we generated a funnel plot, we did not detect definitive evidence of publication bias (see Electronic Supplementary Material).

Second, "before and after" studies, especially within a single ICU, are susceptible to a "Hawthorne effect", whereby practice patterns temporarily change, in part because clinicians know that their practices are being audited.

Third, it is likely that other positive changes were introduced at individual centers over time, apart from implementation of a neurocritical care service. For example, in the study by Samuels and colleagues, there was a clear temporal transition from surgical clip ligation to more widespread use of endovascular coil embolization of cerebral aneurysms. In addition, it is likely that the technical skills and judgment of other clinicians, such as neurosurgeons or neuro-interventionalists, have matured over time.

Finally, it must also be acknowledged that some of the benefits observed by Samuels and colleagues, and by others, may largely reflect the introduction of an intensivistled, systematic, organized approach to critically ill patients, rather than only to specific content expertise in neurocritical care [23]. At least some of the organizational models that neurocritical care units were compared against did not involve the presence of any intensivist. Furthermore, in some cases, the observed improvements in outcomes may have been partially attributable to a transition from an "open" to "closed" ICU.

While the involvement of an intensivist is crucial, the importance of the contributions of other health professionals is becoming increasingly apparent. For example, a recent multi-center RCT found daily physiotherapy to improve functional status at hospital discharge, reduce the
Odds ratio and $95 \% \mathrm{Cl}$

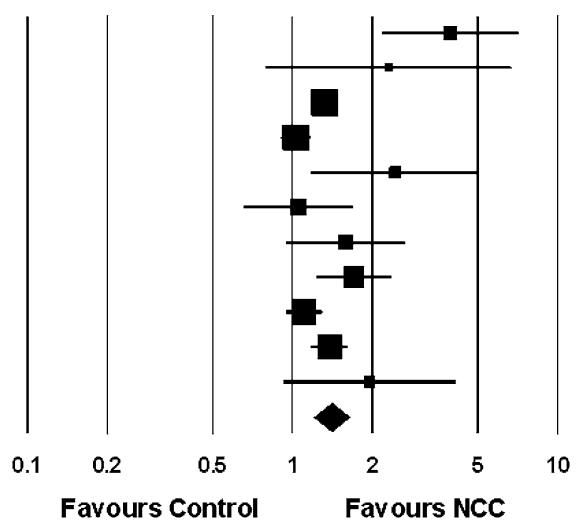

presented separate data for patients with intracranial hemorrhage and ischemic stroke. The size of squares in Forrest plot is proportional to number of patients in study)

duration of mechanical ventilation and ameliorate delirium [24]. Several studies have shown that the presence of a pharmacist on rounds in the ICU is associated with a reduction in adverse drug reactions [25]. Experienced intensivists are well aware of the practical assistance that these and other health professionals provide in patient care.

Do neurocritical care units save lives and improve outcomes? In summary, existing studies have notable limitations, and there is a considerable degree of heterogeneity in the published results. Nevertheless, the cumulative experience, involving almost 25,000 patients, strongly suggests that they do. Although logistical and administrative barriers may exist, reluctance to adopt this model of care should not be based solely on a perception of lack of evidence. However, it seems obvious that the mere presence of a neurointensivist will not lead to improvements in outcomes. It is more likely that concomitant system changes must occur. Unfortunately, published studies do not clarify which specific interventions or modifications in practice were responsible for the observed positive effects. Thus, future research should aim to determine which factors are of particular benefit.

Acknowledgments The authors thank Drs. M. Diringer, E. Keller and D. Seder for providing additional information about their studies.

\section{References}

1. Zygun DA, Kortbeek JB, Fick GH, Laupland KB, Doig CJ. Nonneurologic organ dysfunction in severe traumatic brain injury. Crit Care Med. 2005;33:654-60.

2. Hillman K, Chen J, Cretikos M, Bellomo R, Brown D, Doig G, Finfer S, Flabouris A, MERIT Study Investigators. Introduction 
of the medical emergency team (MET) system: a cluster-randomized controlled trial. Lancet. 2005;365:2091-7.

3. Samuels O, Webb A, Culler S, Martin K, Barrow D. Impact of a dedicated neurocritical care team in treating patients with aneurismal subarachnoid hemorrhage. Neurocrit Care. 2011. doi: 10.1007/s12028-011-9505-z.

4. Warme PE, Bergstrom R, Persson I. Neurosurgical intensive care improves outcomes after head injury. Acta Neurochir. 1991;110: 57-65.

5. Diringer MN, Edwards DF. Admission to a neurologic/neurosurgical intensive care unit is associated with reduced mortality rate after intracerebral hemorrhage. Crit Care Med. 2001;29: $635-40$.

6. Mirski MA, Chang CWJ, Cowan R. Impact of a neuroscience intensive care unit on neurosurgical patient outcomes and cost of care. J Neurosurg Anesthesiol. 2001;13:83-92.

7. Elf K, Nilsson P, Enblad P. Outcome after traumatic brain injury improved by an organized secondary insult program and standardized neurointensive care. Crit Care Med. 2002;30:2129-34.

8. Patel HC, Menon DK, Tebbs S, Hawker R, Hutchinson PJ, Kirkpatrick PJ. Specialist neurocritical care and outcome from head injury. Intensive Care Med. 2002;28:547-53.

9. Suarez JI, Zaidat OO, Suri MF, Feen ES, Lynch G, Hickman J, Georgiadis A, Selman WR. Length of stay and mortality in neurocritically ill patients: Impact of a specialized neurocritical care team. Crit Care Med. 2004;32:2311-7.

10. Varelas PN, Conti MM, Spanaki MV, Potts E, Bradford D, Sunstorom C, Fedder W, Bey LH, Jaradeh S, Gennarelli TA. The impact of a neurointensivist-led team on a semiclosed neurosciences intensive care unit. Crit Care Med. 2004;32:2191-8.

11. Lerch C, Yonekawa Y, Muroi C, Bjeljac M, Keller E. Specialized neurocritical care, severity grade, and outcome of patients with aneurysmal subarachnoid hemorrhage. Neurocrit Care. 2006;5: 85-92.

12. Lott JP, Iwashyna TJ, Christie JD, Asch DA, Kramer AA, Kahn JM. Critical illness outcomes in specialty versus general intensive care units. Am J Respir Crit Care Med. 2009;179:676-83.

13. Josephson SA, Douglas VC, Lawton MT, English JD, Smith WS, Ko NU. Improvement in intensive care unit outcomes in patients with subarachnoid hemorrhage after initiation of neurointensivist co-management. J Neurosurg. 2010;112:626-30.

14. Palminteri J, Duprey J, Rughani A, Tu C, Lin C, McCrum B, Seder D. Effect of hiring a neurointensivist on severity-adjusted ICH mortality. Neurocrit Care. 2010;13:S101.
15. Varelas PN, Eastwood D, Yun HJ, Spanaki M, Bey LH, Kessaris C, Gennarelli TA. Impact of a neurointensivist on outcomes in patients with head trauma treated in a neuroscience intensive care unit. J Neurosurg. 2006;104:713-9.

16. Varelas PN, Schultz L, Conti M, Spanaki M, Genarrelli T, Hacein-Bey. The impact of a neuron-intensivist on patients with stroke admitted to a neuroscience intensive care unit. Neurocrit Care. 2008;9:293-9.

17. Bershad EM, Feen ES, Hernandez OH, Suri MF, Suarez JI. Impact of a specialized neurointensive care team on outcomes of critically ill acute ischemic stroke patients. Neurocrit Care. 2008;9:287-92.

18. Varelas PN, Hacein-Bey L, Schultz L, Conti M, Spanaki MV, Gennarelli TA. Withdrawal of life support in critically ill neurosurgical patients and in-hospital death after discharge from the neurosurgical intensive care unit. Clinical article. J Neurosurg. 2009;111:396-404.

19. Varelas PN, Abdelhak T, Wellwood J, Benczarski D, Elias SB, Rosenblum M. The appointment of neurointensivists is financially beneficial to the employer. Neurocrit Care. 2010;13:228-32.

20. Varelas PN, Spanaki MV, Hacein-Bey L. Documentation in medical records improves after a neurointensivist's appointment. Neurocrit Care. 2005;3:234-6.

21. Helms AK, Torbey MT, Hacein-Bey L, Chyba C, Varleas PN. Standardized protocols increase organ and tissue donation rates in the neurocritical care unit. Neurology. 2004;63:1955-7.

22. Rincon F, Mayer SA, Rivolta J, Stillman J, Boden-Albala B, Elkind MS, Marshall R, Chong JY. Impact of delayed transfer of critically ill stroke patients from the Emergency Department to the Neuro-ICU. Neurocrit Care. 2010;13:75-81.

23. Pronovost PJ, Angus DC, Dorman T, Robinson KA, Dremsizov TT, Young TL. Physician staffing patterns and clinical outcomes in critically ill patients: a systematic review. JAMA. 2002;288: 2151-62.

24. Schweickert WD, Pohlman MC, Pohlman AS, Nigos C, Pawlik AJ, Esbrook CL, Spears L, Miller M, Franczyk M, Deprizio D, Schmidt GA, Bowman A, Barr R, McCallister KE, Hall JB, Kress JP. Early physical and occupational therapy in mechanically ventilated, critically ill patients: a randomized controlled trial. Lancet. 2009;373:1874-82.

25. Leape LL, Cullen DJ, Clapp MD, Burdick E, Demonaco HJ, Erickson JI, Bates DW. Pharmacist participation on physician rounds and adverse drug events in the intensive care unit. JAMA. 1999;282:267-70. 